\title{
Application of Moving-Window based on SCMC in finite element analysis of composite materials
}

\author{
XinHai XIANG ${ }^{1, \text { a }}$, YouCai WANG ${ }^{2, \text { b }}$, HaiQing XIAO ${ }^{3, c}$, GuangZhao YANG ${ }^{4, d}$ \\ ${ }^{1234}$ High-tech Institute, Fan Gong-ting South Street on the $12^{\text {th }}$, Qing Zhou, Shan Dong, China

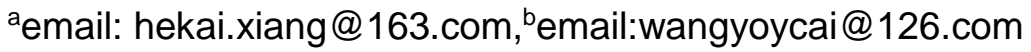

\begin{abstract}
Keywords: Moving-Window, SCMC, composite material, finite element analysis.
Abstract. In this paper, the Moving-Window method based on SCMC is used to calculate the property field of composite materials, and the results are input into the material properties of finite element analysis. By connecting with the finite element analysis program ANSYS, the local stress of non-homogeneous composite material under a given load is calculated. In order to reduce the computational cost and obtain the correct stress distribution in the calculation process, the local area average of the material property field is carried out. The results show that the finite element analysis of the Moving-Window method based on SCMC can conveniently calculate the local stress of non-homogeneous material, and the local stresses maintain the local variability of non-homogeneous composites. Additionally, the local stress calculation shows that there is a shear coupling in the non-homogeneous materials.
\end{abstract}

\section{Introduction}

The composite structure has two characteristics of macro and micro, and its damage is not only related to macro stress and strain field, but also closely related to micro stress and strain field. For stress analysis of composite materials, the existing method is to replace the overall material property with average material properties and composite material is used as homogeneous material for analysis. In fact, composite structure is generally non-homogeneous material, therefore, this substitution introduces the error[1] in calculating the local behavior of non-homogeneous materials. When evaluating the behavior of certain materials, the local response of the material is very important. To obtain the local response of the composite, the finite element method can be used to model the real structure of the composite. However, because of the large difference between macro scale and micro scale, the number of structured grids is huge and the computation efficiency is very low, especially for large complex structures, the establishment and solution calculation time of the finite element model is often too long. As a technique for dealing with random structural models of non-homogeneous materials, the Moving-window[2] technology uses window method combined with micromechanics to obtain the local property field of the material, and then inputs the obtained property field into finite element analysis for local stress analysis. The advantage of this method is that large complex structures can be decomposed by window methods, and avoid the difficulty of solving.

The GMC model proposed by Aboudi[3] is limited in the application of anisotropic material calculation due to the lack of consideration of the shear coupling problem. The Strain-Compatible Method of Cells (SCMC) developed by Hwen Gan[4,5] et al. replaced the stress continuous conditions in the GMC model with stress equilibrium conditions, and the strain compatibility condition is added to make it have shear coupling characteristics, and the calculation efficiency of GMC is basically maintained. In this paper, based on the idea of digital image characterization of composite microstructures, the Moving-Window based SCMC is used to analyze the composite material from the actual micro structure digital image of the composite.

\section{The Moving-Window based on SCMC}

SCMC needs to mesh the microstructure of composite materials, and the mesh generation becomes very difficult for complex fiber shape and arrangement. In this paper, the mesh generation method based on digital image technology is adopted: select the entire arrangement of a region or local 
location of interest in the microscopic image of the non-homogeneous composite material, which obtained from the digital image of the reconstructed composite microstructure by using the Moving-Window [6], the selected area is cut out from the non-uniform image which called the moving window, as a representative cell for the SCMC analysis with the pixel as the cell unit. In this way, the effective properties of the material can be calculated theoretically for many complicated microstructures without considering the division of grids. SCMC predicts the parameters of orthotropic materials in this area based on the shape, arrangement and material properties of the phases contained in the window. Each material property is assigned to the local coordinates of the corresponding window center point, as shown in Figure 1.

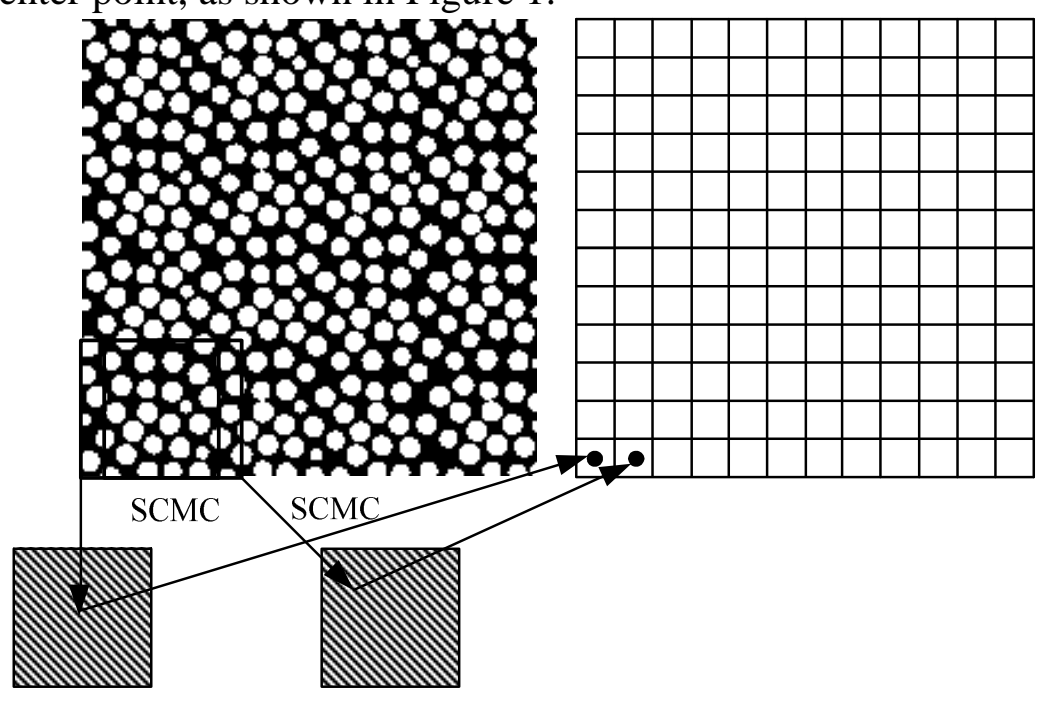

Homogenization Window Homogenization Window

Fig. 1 Moving -Window based on SCMC

The selected moving window has a square $n \times n$ geometry ( $\mathrm{n}$ in pixels), the local homogenization process is repeated over the entire image with the same window size, with each window overlapping the $(n-1)$ pixels of the previously processed window. Therefore, for an $N \times M$ image, $(N-n+1) \times$ $(M-n+1)$ windows can be obtained by the Moving-Window based on SCMC, and the material property field of $(N-n+1) \times(M-n+1)$ windows is also obtained. For concise, the Moving-Window based on SCMC abbreviate as Moving-Window SCMC in the rest of this paper. Specific implementation steps as follows.

Step1: Establish the microstructural model of composite cross section digital image by taking pictures, image processing and image reconstruction of the cross section of the unidirectional long fiber reinforced composite.

Step2: In the established micro structure model, use the moving window to select a local area as a window, and calculate the window with SCMC to obtain the material property.

Step3: Move the window and homogenize the whole microstructure, calculate the elastic property field coefficient of variation, random field distribution and correlation coefficient to obtain the property field of the material, and the results are input to the material properties of finite element analysis.

\section{The establishment and connection of the finite element analysis model}

According to the material property field generated by the Moving-Window SCMC, the cross section of the non-homogeneous composite sample is simulated by the finite element program ANSYS, and two kinds of finite element models are generated. One is the window size model based on the total sample size $5 \% \times 5 \%$, and the other is the window size model based on the total sample size $10 \% \times 10 \%$.

Since in the homogenization process, each moving window overlaps the previous window $(n-1)$ pixels. In the 100 pixels $\times 100$ pixels microstructure sample model, the windows of 5 pixels $\times 5$ pixels get $96 \times 96$ partial uniform windows and 10 pixels $\times 10$ pixels get $91 \times 91$ partial uniform windows , 
and two windows of different sizes were used to illustrate the effect of different window sizes on the local response of materials. The boundary conditions of the model can be expressed by the following equation.

$$
\left\{\begin{array}{l}
\sigma_{x x}(x=0.0)=0.0 \\
\sigma_{x x}(x=1.0)=1.0 \\
\sigma_{y y}(y=0.0)=\sigma_{y y}(y=1.0)=0.0 \\
\tau_{x y}(x=0.0)=\tau_{x y}(x=1.0)=0.0 \\
\tau_{y x}(y=0.0)=\tau_{y x}(y=1.0)=0.0 \\
u_{x}(x=0.0)=0
\end{array}\right.
$$

The finite element model is meshed by a plane Stress Element with two degrees of freedom per node. It is noted that in calculating material behavior, both planar stress and plane strain assumption do not actually produce significant qualitative difference. Here, the stiffness matrix of two-dimensional element is calculated from the standard finite element method [7].

$$
\mathbf{K}^{(e)}=\int_{A(e)} \mathbf{B}(x, y)^{T} \mathbf{D}^{(e)} \mathbf{B}(x, y) d A
$$

Where $\mathbf{B}(x, y)$ is the strain-displacement matrix of the corresponding element $(e), A^{(e)}$ is the area of the element, and $\mathbf{D}^{(e)}$ is the constitutive matrix of the plane stress orthotropic material, which is defined as:

$$
D^{(e)}=\left[\begin{array}{ccc}
\frac{1}{E_{x x}^{(e)}} & \frac{v_{y x}^{(e)}}{E_{y y}^{(e)}} & 0 \\
\frac{v_{x y}^{(e)}}{E_{x x}^{(e)}} & \frac{1}{E_{y y}^{(e)}} & 0 \\
0 & 0 & \frac{1}{G_{x y}^{(e)}}
\end{array}\right]
$$

Where $E_{y y}^{(e)}$ and $E_{x x}^{(e)}, v_{y x}^{(e)}$, and $G_{x y}^{(e)}$ are the transverse elastic modulus, the transverse Poisson's ratio, and the transverse shear modulus of the corresponding element $(e)$, respectively.

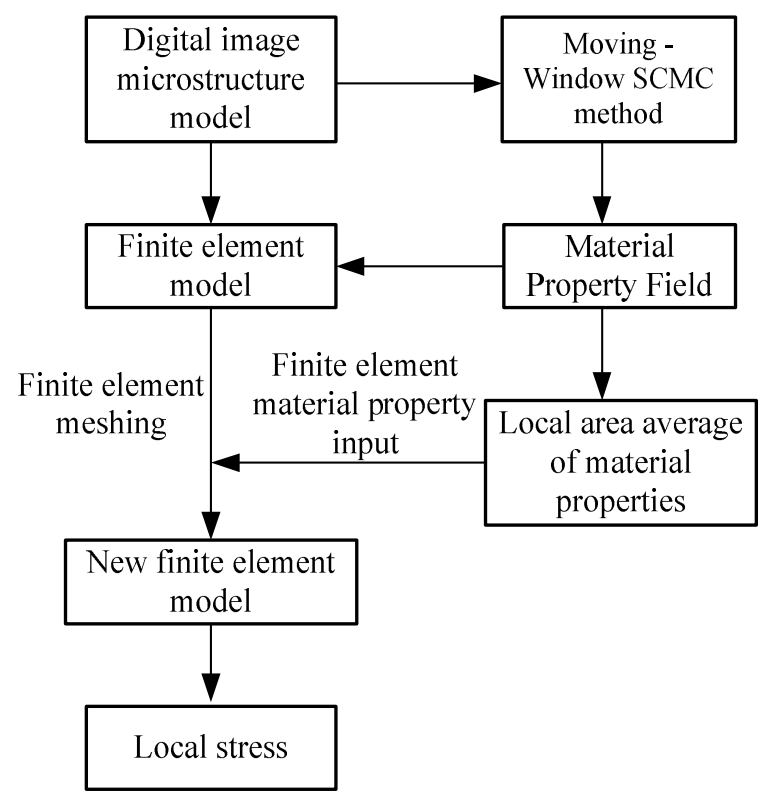

Fig. 2 Finite element program flow of Moving-Window SCMC 
In this paper, the finite element program ANSYS is used to solve various complex structural problems. When installing the ANSYS program choose to install the Customization Tools, it provides a FORTRAN interface subprogram that can be modified. The Moving- Window SCMC is integrated into the finite element program through the interface program provided by ANSYS. The program processing process is shown in Figure 2.

\section{Finite Element Analysis of Moving-Window SCMC for Composite Materials}

The material properties of each uniform window can be obtained from the Moving-Window SCMC, but when the size of the sample is too large, the calculation cost is too high. In order to obtain a reliable stress distribution, and which is also reasonable for computational efficiency, it is need to redefine the mesh size and analyse the convergence of meshing. In this paper, 6 mesh sizes are considered based on 100 pixels $\times 100$ pixels non-homogeneouscomposite material. When the window size is 5 pixels $\times 5$ pixels, the mesh sizes are: $4 \times 4,8 \times 8,16 \times 16,32 \times 32,48 \times 48$ and $96 \times 96$; When the window size is 10 pixels $\times 10$ pixels, the mesh sizes are: $3 \times 3,7 \times 7,15 \times 15,30 \times 30,45 \times 45$ and 91 $\times 91$. Material properties specified for each newly generated finite element unit which are obtained by local area averaging of the material properties that obtained from the Moving-Window SCMC method [8].

$$
C^{(e)}=\frac{1}{A^{(e)}} \int_{A(e)} C_{0}(x, y) d x d y
$$

Here $C^{(e)}$ is the material property assigned to each finite element $(e), C_{0}(x, y)$ is the material property of each uniform window obtained from the Moving-Window SCMC, and $A^{(e)}$ is the area of the finite element $(e)$. It is need to note that the material property of the local area average obtained by Eq. 4 is only an approximation process, more precise processing should be performed by averaging the micro-mechanical analysis of each newly generated finite element sub-model. Figure 3 below represents the material field, and the black squares in the figure represent sub-models that are used as a single finite element unit.

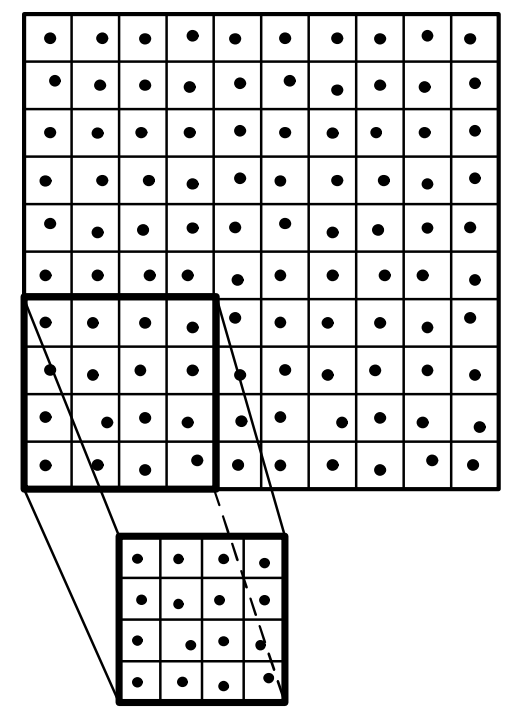

Single finite element model

Fig. 3 Re-meshing finite element model

Micromechanics analysis of the new finite element sub-model can continue to use the SCMC method. Because of the finite element sub-model contains several different material property results units (i.e., each window) obtained from the Moving-Window SCMC. Therefore, these units with different properties can be used as each phase in the SCMC analysis of multiphase composites, and the entirety of these units is a single cell for SCMC analysis of multiphase composites. By 
homogenizing the SCMC multiphase composite material, the equivalent material properties can be assigned to the equivalent material properties of the newly generated finite element unit. In this paper only uses the partial area average of Eq. 4 . For $5 \% \times 5 \%$ and $10 \% \times 10 \%$ moving window sizes, when the finite element meshes are $48 \times 48$ and $45 \times 45$, Figures 4,5 , and 6show the stress nephogram of $\sigma_{x x}$, $\sigma_{y y}$, and $\sigma_{x y}$, respectively.
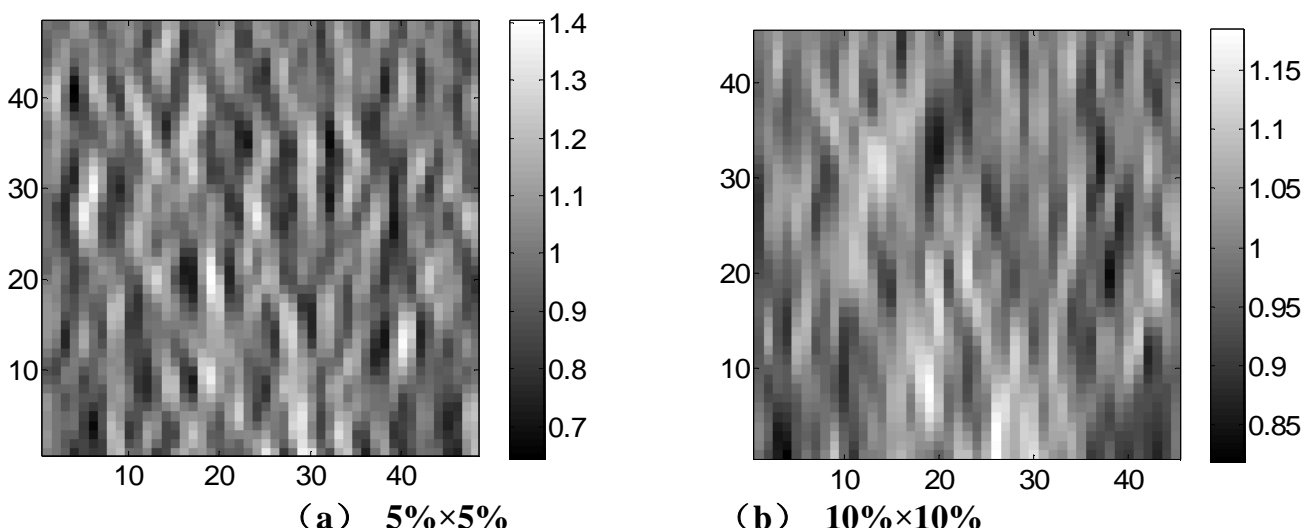

(b) $10 \% \times 10 \%$

Fig. $4 \sigma_{x x}$ stress nephogram under different window size
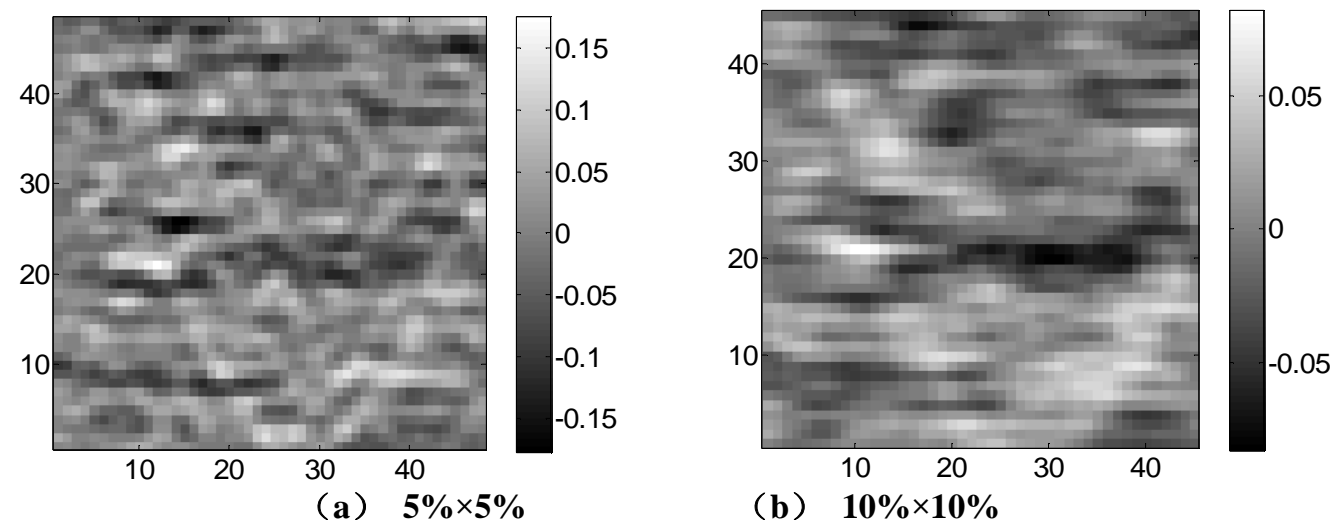

Fig. $5 \sigma_{y y}$ stress nephogram under different window size
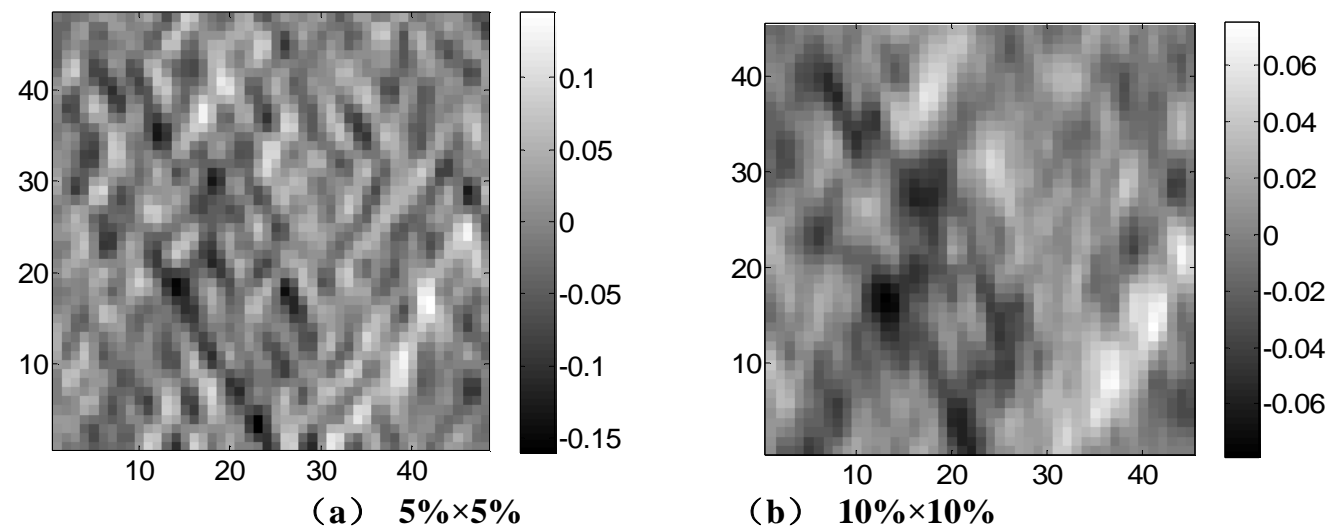

Fig. $6 \sigma_{x y}$ stress nephogram under different window size

As can be seen from the stress nephogram of the entire sample above, the calculated results have considerable variability. For example, for a 5\% x 5\% moving window, the Transverse positive stress $\sigma_{x x}$ ranges from a maximum of 1.46 to a minimum of 0.63 . It shows that in some local area, the maximum value of the Transverse positive stress is $46 \%$ larger than the average value obtained by macroscopic analysis, and the minimum value is $37 \%$ smaller than the macroscopic average. The transverse stress $\sigma_{y y}$ and shear stress $\sigma_{x y}$ also have the same effect, the ranges of $\sigma_{y y}$ and $\sigma_{x y}$ ranged from 0.18 to -0.20 and 0.18 to -0.18 respectively. If the mean total error response is taken into account, the average of stress is 0 . It can also be seen that these stress contour maps show local shear 
coupling in transverse loading. And these stress clouds at different window sizes indicate that window size has a significant impact on the form and extent of local stress variations.

\section{Conclusions}

The Moving-Window based on SCMC is used to homogenize the composite cross-section microstructure model. After generating the elastic property field of the non-homogeneous material, in order to determine the local stress and strain of the non-homogeneous material microstructure under a given loading condition, the material property field is used as an input to the finite element analysis. The local stress distribution of the non-homogeneous composite was calculated by finite element analysis which local stress maintains local variability. At the same time, the local stress calculation shows that there is a shear coupling in the local non-homogeneous material. Finite element analysis of different window sizes also illustrates the importance of choosing the right window size.

\section{References}

[1] Frantziskonis, G.. N.. Stochastic Modeling of Heterogeneous Materials-A Process for the Analysis and Evaluation of Alternative Formulations, Mechanics of Materials, 1998, 27: 165-175.

[2] Baxter, S .C. \& Graham, L. L.. Characterization of Random Composites Using Moving Window Technique, ASCE Journal of Engineering Mechanics, 2000, 126(4):389-404..

[3] Aboudi J. Micromechanical analysis of composites by the method of cell-update. Appl Mech Rev 1996, 49(10):583-591.

[4] Carlos E. Orozco, Hewen Gan. Viscoplastic response of multiphase composites using a strain-compatible volume-averaging method. Composites: Part B, 2002(33), 301-313.

[5] Hewen Gan, Carlos E. Orozco, Carl T. Herakovich. A strain-compatible method for micromechanical analysis of multi-phase composites. International Journal of Solids and Structures, 2000(37), 5097-5122.

[6] LI Haibin,ZHU Shijie.Application of Moving - Window in Characterization of Composite Materials Elasticity Property Field.FIBER COMPOSITES.2009(2):3-8.

[7] Hughes TJR. The finite element method. Englewood Cliffs, NJ: Prentice Hall, 1987.

[8] Graham L. L., Baxter S. C. Simulation of local material properties based on moving-window GMC. Probab Engng Mech. 2001, 16(4):295-306. 